\title{
Doping composite of polyaniline and reduced graphene oxide with palladium nanoparticles for room-temperature \\ hydrogen-gas sensing
}

\author{
Yongjin Zou, ${ }^{\mathrm{a}, \mathrm{b}, \mathrm{c}}$, Qingyong Wang ${ }^{\mathrm{a}, \mathrm{b}}$, Cuili Xiang ${ }^{\mathrm{a}, \mathrm{b}, *}$, Chengying Tang ${ }^{\mathrm{a}, \mathrm{b}}$, Hailiang \\ $\mathrm{Chu}^{\mathrm{a}, \mathrm{b}}$, Shujun Qiu, ${ }^{\mathrm{a}, \mathrm{b}}$, Erhu Yan ${ }^{\mathrm{a}, \mathrm{b}}$, Fen Xu ${ }^{\mathrm{a}, \mathrm{b}}$, Lixian $\operatorname{Sun}^{\mathrm{a}, \mathrm{b}, *}$
}

${ }^{\mathrm{a}}$ Guangxi Key Laboratory of Information Materials, Guilin University of Electronic Technology,

Guilin 541004, P.R. China

${ }^{\mathrm{b}}$ Guangxi Collaborative Innovation Center of Structure and Property for New Energy Materials,

Guilin 541004, P.R. China

${ }^{\mathrm{c}}$ Guangxi Experiment Center of Information Science, Guilin University of Electronic Technology,

Guilin 541004, P.R. China

*Corresponding author. Fax: 86-773-2216607 (C.L. Xiang), 86-773-2303763 (L.X.

$\underline{\text { Sun). }}$.

E-mail address: xiangcuili@guet.edu.cn (C.L. Xiang), sunlx@guet.edu.cn (L.X. Sun). 


\section{ABSTRACT}

A composite consisting of polyaniline (PANI) and reduced graphene oxide (rGO) was synthesized by in situ polymerization of the monomer aniline in the presence of $\mathrm{rGO}$ under acidic conditions. The PANI-rGO composite substrate was doped with $\mathrm{Pd}$ nanoparticles via chemical reduction. The resulting Pd-PANI-rGO nanocomposite was characterized by transmission electron microscopy, scanning electron microscopy, X-ray diffraction, and Fourier-transform infrared spectroscopy before it was utilized to fabricate a hydrogen sensor. Compared with the sensor based on PANI-rGO or PANI, the Pd-PANI-rGO sensor was highly sensitive and selective to hydrogen gas, with fast response time in air at room temperature. The significantly enhanced sensitivity resulted from the faster spill-over effect, dissociation of hydrogen molecules on Pd, and the high surface area of the PANI-GO composite. Based on its improved sensing properties, ease of fabrication, and stable operation, the Pd-PANI-rGO nanocomposite shows promise for high-performance hydrogen-sensing applications.

Keywords: Pd nanoparticles; Graphene; Polyaniline; Hydrogen sensor; Nanocomposite 


\section{Introduction}

Graphene, a very attractive two-dimensional carbon nanomaterial with superior electrical conductivity, excellent mechanical flexibility, and high thermal and chemical stability, has been widely used in many fields such as integrated electronics and optoelectronics [1-3]. Through wet-chemical methods, graphene can be simply made into either graphene oxide (GO) or reduced graphene oxide (rGO). Compared with raw graphene, oxidized graphene is hydrophilic and has good dispersion in water, both of which are important criteria for solution processing and further modification [4]. Meanwhile, these oxygen-containing groups enable graphene sheets to strongly interact with small polar molecules or polymers to form graphene-intercalated or exfoliated composites [5]. However, pristine GO is not suitable for gas-sensing applications because of the low adsorption energies of gas molecules on the GO surface [6]. In addition, the selectivity of a graphene-based gas sensor must be improved for each target gas. To overcome this drawback, functionalization of GO with metal particles, a polymer, or a metal oxide is considered to be an effective way to improve the gas-sensing capability of GO because of the synergistic effects of GO and the additive [7-10].

In recent years, the synthesis and application of conducting polymers have attracted great interest in the scientific community [11-13]. Among various polymers that have been developed, polyaniline (PANI) is one of the promising candidates for gas sensing [14-17]. PANI has good thermal and electrochemical stability, a unique conducting mechanism, unusual doping and dedoping chemistry, and low cost, which 
lead to potential application in many fields such as battery electrodes, supercapacitor, gas sensors, and electrocatalytic devices [18-20]. Moreover, PANI has the highest environmental stability and is recognized as the only conducting polymer that is stable in air. PANI has been utilized as a sensor material because it is sensitive at room temperature, and the application of PANI-based sensors has been expanded to the detection of a range of gases by combining PANI with other nanomaterials [21-25]. The incorporation of rGO in a polymer is a good way to exploit the advantages of both materials while greatly improving the thermal stability and electrical properties of the polymer [26-28]. In addition, a large number of polymer-rGO nanocomposites have become accessible in the form of end-functionalized derivatives because of their small particle size and intercalation properties [29]. Therefore, the gas-sensing potential of polymer-GO nanocomposites has attracted much recent research attention [30-32]. In particular, PANI-based sensors have been shown to exhibit ultrahigh sensitivity, fast response, satisfying reversibility, etc., and they are suitable for practical applications $[33,34]$.

To the best of our knowledge, no attempt has been made until our current study to examine the performance of a conducting-polymer-graphene composite decorated with metal nanoparticles. Therefore, it is a significant body of work that explores the fabrication of a $\mathrm{H}_{2}$ gas sensor based on graphene-PANI nanocomposites and investigates the properties of the nanocomposites and performance of the sensor. In this study, different mass concentrations of rGO were optimized to improve the detection performances. Infrared, morphological, and electrical characterizations of 
the graphene-PANI thin films were also carried out.

\section{Experimental}

\subsection{Materials}

Aniline, ammonium persulfate (APS), cetyltrimethylammonium bromide (CTAB), $\mathrm{K}_{2} \mathrm{PdCl}_{6}$, and indium-tin oxide (ITO; surface resistivity: 70-100 $\Omega /$ sq) glass were obtained from Sigma-Aldrich, USA. Reduced graphene oxide $(>99.5 \%)$, with a thickness of 4-20 nm and a diameter of $0.5-10 \mu \mathrm{m}$, was supplied by Chengdu Organic Chemicals Co. Ltd, China. All chemicals and reagents were used as received, except for aniline, which was distilled under reduced pressure before use.

\subsection{Preparation of $P d-P A N I-r G O$}

The PANI-rGO composites were prepared according to established procedures. In brief, $0.15 \mathrm{~g}$ of $\mathrm{rGO}$ was dispersed in $100 \mathrm{~mL}$ of a $0.01 \mathrm{M} \mathrm{HCl}$ solution and ultrasonicated for $2 \mathrm{~h}$ to form a uniform suspension; $0.5 \mathrm{~mL}$ of aniline was then added to the suspension and ultrasonicated for $1 \mathrm{~h}$ before polymerization. Next, $0.5 \mathrm{~g}$ of APS was dissolved in $20 \mathrm{~mL}$ of deionized water and slowly added drop by drop to the prepared suspension from a burette. After stirring for $4 \mathrm{~h}$, the solution was filtered and washed thoroughly with deionized water and methanol, and then dried in an oven at $60{ }^{\circ} \mathrm{C}$ under vacuum. Different concentrations of rGO were employed in our research. For comparison, PANI was also prepared under the same conditions.

The PANI-rGO composite was doped with Pd nanoparticles as follows: First, $1 \mathrm{mg}$ 
of $\mathrm{K}_{2} \mathrm{PdCl}_{6}$ was added to $5 \mathrm{~mL}$ of deionized water (density: $0.2 \mathrm{~g} / \mathrm{L}$ ); $0.1 \mathrm{~g}$ of the PANI-rGO powder was then dispersed in the solution under sonication for $2 \mathrm{~h}$. Next, $1 \mathrm{~mL}$ of a $\mathrm{NaBH}_{4}$ solution (density: $1 \mathrm{~g} / \mathrm{L}$ ) was added drop-wise to the mixture with vigorous stirring for $20 \mathrm{~min}$. The mixed solution was then dried at $60{ }^{\circ} \mathrm{C}$ under vacuum for $10 \mathrm{~h}$. Fig. 1 shows a schematic diagram of the preparation of Pd-PANI-rGO.

\subsection{Sensor fabrication and sensing tests}

The $\mathrm{H}_{2}$ sensor was fabricated on an ITO layer deposited on a glass substrate. Before the fabrication, the sheets of ITO/glass were sonicated in ethanol, washed with deionized water, and then dried in a nitrogen flow. Copper foil tape with conductive adhesive on one side was purchased from Shanghai Mingyu Metal Materials Company Inc., China. The electrical contacts were made by attaching two copper foils (with a 5-mm separation) onto the ITO surface. The effective sensing area was $0.5 \times 3$ $\mathrm{cm}^{2}$. Next, $0.1 \mathrm{~g}$ of the Pd-PANI-rGO powder was suspended in $5 \mathrm{~mL}$ of an ethanol solution under sonication for 30 min to produce a uniform suspension, after which 1 $\mathrm{mL}$ of the suspension was drop-coated onto the gap between the two copper foils by a syringe and dried in air. The thickness of the thin film, measured by SEM, was about 2.1 $\mu \mathrm{m}$. The sensor fabrication process is illustrated in Fig. 2.

The sensor testing was carried out in a laboratory-constructed, airtight chamber by exposing the prepared sensor to various concentrations of $\mathrm{H}_{2}$ gas. To measure the gas response, the sensor was introduced into the test chamber (volume: $0.5 \mathrm{~L}$ ) with gas 
inlet and outlet ports. The gas chamber was purged with $\mathrm{N}_{2}$ for $30 \mathrm{~min}$ before the test. Standard $\mathrm{H}_{2}(1 \mathrm{vol} \%)$ and air were injected into the chamber. The pressure of the chamber was $1.2 \mathrm{~atm}$, and the flow rate of the synthetic gas was $100 \mathrm{sccm}$. The electrical resistance of the thin film was measured by a multimeter (Keithley 2700, USA) that was connected to a computer for data acquisition. For the selectivity experiment, a known gas $\left(\mathrm{CH}_{3} \mathrm{OH}, \mathrm{CO}_{2}\right.$, or $\left.\mathrm{H}_{2} \mathrm{~S}\right)$ with a particular concentration was injected through a syringe. The gas-testing setup is illustrated in Fig. 3. The sensor response $S$ to $\mathrm{H}_{2}$ gas is defined by the following equation:

$$
S(\%)=\frac{\Delta R \times 100}{R_{\text {air }}}=\frac{\left(R_{G}-R_{\text {air }}\right) \times 100}{R_{\text {air }}}
$$

where $R_{\mathrm{G}}$ and $R_{\text {air }}$ are the electrical resistances of the sensitive films exposed to $\mathrm{H}_{2}$ and dry air, respectively. The response time is defined as the time required for the sensor to reach $90 \%$ of the change in total resistance, and the recovery time is the time required for the sensor to return to $10 \%$ of the value before exposure to a gas. All experiments were carried out at room temperature $\left(25 \pm 1^{\circ} \mathrm{C}\right)$ and a relative humidity of $42 \pm 3 \mathrm{RH} \%$.

\subsection{Instruments and analysis}

The morphology of the nanocomposites was characterized by scanning electron microscopy (Quanta 200, FEI, USA) at an electron voltage of $10 \mathrm{kV}$. Fourier-transform infrared (FTIR) spectra were recorded by a Nicolet 6700 spectrometer (Thermo Scientific, USA), using $\mathrm{KBr}$ pellets. The crystalline phases were characterized with X-ray diffraction (XRD) on a diffractometer (1820, Philips 
Analytical, The Netherlands) with $\mathrm{Cu} \mathrm{K}_{\alpha}$ radiation $(\lambda=1.5418 \AA)$. The surface of the sample was coated with gold before SEM analysis. The specific surface areas (SSAs) of the catalysts were measured by obtaining $\mathrm{N}_{2}$ adsorption isotherms at $-196^{\circ} \mathrm{C}$ using the Brunauer-Emmett-Teller (BET) method (Autosorb iQ2, Quantachrome, USA). The samples were degassed at $423 \mathrm{~K}$ until the vacuum pressure was below $10^{-4} \mathrm{~Pa}$. An average value was obtained by taking three measurements for each sample.

\section{Results and discussion}

\subsection{Characterization of $P d-P A N I-r G O$ nanocomposites}

An SEM was used to investigate the surface morphology of the samples. In the SEM image of rGO in Fig. 4a, it can be seen that the rGO surface was wrinkled, which is a typical feature of exfoliated rGO. The exfoliation revealed sufficient functional groups (such as hydroxyl and carboxylic acids) on the surface, which potentially provided active sites for anchoring external nanoparticles [5]. The pure PANI consisted of random short-rod agglomerates with a diameter of $\sim 80 \mathrm{~nm}$ (Fig. 4b). The PANI-rGO composites produced by in situ chemical polymerization under typical conditions exhibited layered and fibrous structures (Fig. 4c), indicating that the rGO sheets were homogeneously surrounded by PANI nanofibers. After the chemical reduction, Pd nanoparticles with a diameter of $\sim 100 \mathrm{~nm}$ were well-dispersed on the PANI-rGO substrate (Fig. 4d). Pd is an ideal material for hydrogen sensing because of its high sticking coefficient, higher diffusion coefficient, and high selectivity for hydrogen. The well-dispersed Pd nanoparticles promoted faster gas 
response by producing active sites, which is regarded as a form of electronic sensitization [5]. However, lattice expansion and contraction during hydrogenation and dehydrogenation made Pd susceptible to mechanical and topographical instability [35]. Furthermore, the agglomeration of metal nanoparticles in the films resulted in poor sensing performance. Thus, dispersion of nanoparticles on other nanostructures with large surface area would prevent agglomeration. In the current study, the PANI-rGO substrate was well doped with Pd nanoparticles through chemical reduction. The nanocomposites had large surface areas, which were beneficial for hydrogen sensing.

The chemical compositions of the prepared nanocomposites were characterized by FTIR spectra. Fig. 5 shows the FTIR spectra of rGO, PANI, PANI-rGO, and Pd-PANI-rGO. In the spectrum of rGO, the absorption peaks at 3373, 1591, and 1055 $\mathrm{cm}^{-1}$ are attributed to $\mathrm{OH}, \mathrm{C}-\mathrm{O}$ in $\mathrm{COOH}$, and $\mathrm{C}-\mathrm{O}$ in $\mathrm{COH} / \mathrm{COC}$ (epoxy) functional groups, respectively. The broad peak at $3373 \mathrm{~cm}^{-1}$ is attributed to the hydrogen bond between oxygen and hydrogen, which is in agreement with reported data [5]. The peaks at $1587,1483,1294$, and $795 \mathrm{~cm}^{-1}$ in the PANI spectrum can be assigned to the $\mathrm{C}=\mathrm{C}$ stretching vibration of quinoid, $\mathrm{C}=\mathrm{C}$ stretching vibration of benzene rings, stretching vibration of $\mathrm{C}-\mathrm{N}$, and out-of-plane bending of $\mathrm{C}-\mathrm{H}$, respectively [5]. The strong characteristic band at $1140 \mathrm{~cm}^{-1}$ is considered to be a measure of delocalization of electrons, which is ascribed to the characteristic peak of PANI conductivity [36]. These peaks can also be found in the FTIR spectra of the PANI-rGO and Pd-PANI-rGO nanocomposites, confirming that the rGO sheets were covered with 
PANI. However, the peaks at 1604 and $2925 \mathrm{~cm}^{-1}$ in the spectrum of pure PANI-rGO are shifted to 1587 and $2921 \mathrm{~cm}^{-1}$, respectively, in the spectrum of the Pd-PANI-rGO composite. These band shifts to lower wavenumbers may have been caused by the relatively higher protonated state [37].

The crystal structures of the as-prepared samples were characterized by XRD, and the XRD patterns of rGO, PANI, PANI-rGO, and Pd-PANI-rGO are shown in Fig. 6. The pattern of rGO exhibits a (002) diffraction peak at $2 \theta=26.3^{\circ}$, which is also found in the diffraction patterns of PANI-rGO and Pd-PANI-rGO, indicating that rGO was incorporated in the composite. However, with the formation of PANI-rGO, this peak shifts toward a slightly higher $2 \theta$, probably because of the intercalation of PANI chains between the rGO sheets [38]. The spectrum of pure PANI includes a broad peak in the region of $2 \theta=20-30^{\circ}$ and three weak peaks at $2 \theta=24.7^{\circ}, 26.5^{\circ}$, and $29.1^{\circ}$, which indicate the polymer's highly pronounced oscillation structure $[16,34]$. The upper-most line in Fig. 6 shows the XRD pattern of the as-obtained Pd-PANI-rGO. The diffraction peaks at $2 \theta$ of $20-30^{\circ}$ can be assigned to PANI. The diffraction peaks at $2 \theta=39.2^{\circ}, 45.1^{\circ}$, and $68.4^{\circ}$ are associated with the (111), (200), and (220) facets, respectively, of the Pd crystal [6], indicating that the Pd-PANI-rGO nanocomposite was successfully prepared.

To further check the surface areas of these materials, nitrogen adsorption-desorption isotherms were obtained, and the results are shown in Fig. 7. The BET surface areas were $22.4,38.5,44.3$, and $71.5 \mathrm{~m}^{2} \mathrm{~g}^{-1}$ for rGO, PANI, PANI-rGO, and Pd-PANI-rGO, respectively, which indicate that the addition of rGO 
to PANI increased the surface area of the PANI-rGO composite from that of PANI alone. The isotherm of Pd-PANI-rGO is of type IV with a large hysteresis loop, attributed to the abundance of mesopores inside the material [39]. The differences in surface areas and pore size distributions of these materials may be attributed to the different nanoparticle sizes, interparticle spacings, and internal voids. The Pd-PANI-rGO nanocomposite exhibited higher surface area than those of rGO, PANI, and PANI-rGO because the introduction of $\mathrm{rGO}$ was favorable for reducing self-aggregation of PANI and Pd. The mesoporous structures were easily accessible to gas molecules, leading to an increase in adsorption sites on the surface for hydrogen molecules.

\subsection{Hydrogen-sensing properties}

Hydrogen-sensing tests were conducted in ambient air at room temperature $\left(25^{\circ} \mathrm{C}\right)$. Sensors were fabricated from Pd-PANI-rGO nanoparticles, and their hydrogen-sensing performance was evaluated. For comparison, sensors based on PANI and PANI-rGO were also fabricated. The conductivity of the Pd-PANI-rGO nanocomposite decreased with increasing hydrogen concentration. Upon exposure to hydrogen, the adsorption of hydrogen on Pd nanoparticles triggered the formation of Pd hydride. The adsorbed molecules on a nanoparticle's surface and $\mathrm{H}$ species incorporated in the octahedral interstitial sites of the Pd alloy acted as electron scattering centers and decreased the carrier mobility. This caused a hydrogen-induced increase in the electrical resistance of the Pd nanoparticles. For the doped PANI-rGO 
composite, the chemically formed PANI-rGO layer is considered to exhibit p-type behavior [40]. Owing to the difference between the work function of Pd nanoparticles and the charge-neutrality level of PANI-rGO, electrons were transferred from PANI-rGO to the Pd nanoparticles, resulting in a modified charge-neutrality level of the Pd-PANI-rGO interface [35]. Owing to the lower work function of $\mathrm{PdH}_{x}$ in the hydrogen-containing atmosphere, the charge-neutrality level would increase with a decrease in the number of electrons migrating from PANI-rGO to $\mathrm{PdH}_{x}$. The presence of more electrons at the PANI-rGO surface resulted in compensation for its p-type nature and an increase in the electrical resistance [41]. In the absence of hydrogen, the resistance decreased sharply and regained its steady-state value.

The responses of the PANI, PANI-rGO, and Pd-PANI-rGO nanoparticles to $1 \%$ of $\mathrm{H}_{2}$ are shown in Figs. 8 and S1. PANI and PANI-rGO showed weak response to hydrogen gas, suggesting that these two materials by themselves were not sensitive to 1 vol\% hydrogen at room temperature. PANI even shows slightly negative response to hydrogen, which has also been reported in a previous work [29]. On the other hand, Pd-decorated PANI-rGO showed much better hydrogen-sensing performance when compared with PANI-rGO and PANI, exhibiting $\sim 25 \%$ increase in electrical resistance upon exposure to hydrogen at room temperature. The most probable reason was the higher specific surface area of the Pd-PANI-rGO nanocomposite film, which significantly enhanced gas diffusion because of its larger exposure area and penetration depth for hydrogen molecules. Another factor was that Pd has the ability to absorb a large amount of hydrogen at room temperature (about 900 times of its own 
volume), which means the Pd particles were also able to act as a "hydrogen collectors" and were expected to greatly increase the sensitivity of the Pd-PANI-rGO film.

Figs. 9 and S2 show the responses of the Pd-PANI-rGO thin film to hydrogen gas in various concentrations. It is clear that the Pd-PANI-rGO thin film exhibited fast and sensitive response to high-concentration hydrogen; the response also increased linearly as the gas concentration was increased from 0.01 to 2 vol\% (Fig. 9b). The lowest detection limit was $0.01 \mathrm{vol} \%$, which is lower than the result reported for a hydrogen sensor consisting of a composite of Pd nanoparticles and graphene (0.02\%) [15], and the result reported for another sensor consisting of single-walled carbon nanotubes (SWCNTs) loaded with Ni-Pd $(0.2 \%)$ [42]. These results indicate that the Pd-PANI-rGO-based hydrogen sensor was highly sensitive; it could detect hydrogen gas at a concentration much lower than the "lower explosive limit (4 vol\%)" with relatively high sensitivity, suggesting that it could be used in practical applications like hydrogen-leak monitoring.

The response time and recovery time of the Pd-PANI-rGO-based sensor to $1 \%$ hydrogen $\mathrm{H}_{2}$ were 20 and $50 \mathrm{~s}$, respectively. For the PANI-rGO sensing film, the response time was about $120 \mathrm{~s}$ and the recovery time was about $20 \mathrm{~s}$. These results suggest that the $\mathrm{H}_{2}$ sensor based on Pd-PANI-rGO exhibited much stronger and faster response than that of the PANI-rGO sensor. Table 1 compares the $\mathrm{H}_{2}$-sensing properties of various materials reported in the literature with those of Pd-PANI-rGO prepared in this study. It is clear that the Pd-PANI-rGO thin film in our study showed excellent performance in $\mathrm{H}_{2}$-gas detection. 
Different concentrations of rGO were investigated for the preparation of the PANI-rGO composite. The response of the sensor at different rGO concentrations was investigated. As shown in Fig. 10, the response of the sensor to 1 vol\% hydrogen was enhanced by increasing the concentration of $\mathrm{rGO}$, and the response reached a maximum value at $0.15 \mathrm{~g}$. The hydrogen response was reduced when the concentration of rGO was further increased, probably because of the low adsorption energy of the gas molecules on the rGO surface [41].

Since the response of the hydrogen sensor was highly influenced by the amount of doping Pd nanoparticles, the effect of the Pd nanoparticle density on the response of the $\mathrm{H}_{2}$ sensor was investigated by changing the concentration of $\mathrm{K}_{2} \mathrm{PdCl}_{6}$ for chemical reduction. As shown in Fig. 11, the response of the $\mathrm{H}_{2}$ sensor increased with increasing concentration of $\mathrm{K}_{2} \mathrm{PdCl}_{6}$ and reached a maximum value at $0.2 \mathrm{~g} / \mathrm{L}$, after which the response tended to decrease with further increase in the concentration of $\mathrm{K}_{2} \mathrm{PdCl}_{6}$. This can be explained by the tendency for Pd nanoparticles to aggregate when the concentration of $\mathrm{K}_{2} \mathrm{PdCl}_{6}$ was high. The optimized concentration of $\mathrm{K}_{2} \mathrm{PdCl}_{6}$ for Pd doping was $0.2 \mathrm{~g} / \mathrm{L}$, which was chosen for the preparation of Pd-PANI-rGO.

Fig. 12 shows that the hydrogen response varied with time when the Pd-PANI-rGO nanocomposite sensor was exposed to 1 vol $\%$ hydrogen at different temperatures. In the temperature range of 25 to $200{ }^{\circ} \mathrm{C}$, the sensor exhibited improved response when exposed to $1 \%$ hydrogen. This result indicates that the $\mathrm{H}_{2}$-sensing performance of Pd-PANI-rGO was affected by the working temperature because the surface reaction was thermally activated when the temperature increased, which is in agreement with 
the result of a previous report [35]. The effects of temperature on the response time and recovery time were also investigated. As shown in Fig. 13, increasing the operating temperature resulted in faster response and recovery times. The response time decreased from 20 to $10 \mathrm{~s}$ and the recovery time from 50 to $32 \mathrm{~s}$ as the temperature was increased from 25 to $200{ }^{\circ} \mathrm{C}$. This was most likely due to the faster reaction rate of adsorbed and desorbed hydrogen atoms in the active sites at higher temperatures.

The selectivity of the sensor for hydrogen over $\mathrm{CH}_{3} \mathrm{OH}, \mathrm{CO}_{2}$, and $\mathrm{H}_{2} \mathrm{~S}$ was also tested, and the response of the sensor to these gases at the same concentration ( $1 \mathrm{vol} \%)$ is shown in Fig. 14. The sensor did not respond to $\mathrm{CO}_{2}$ and $\mathrm{H}_{2} \mathrm{~S}$, but it responded to 1 vol $\% \mathrm{CH}_{3} \mathrm{OH}$, with a slight improvement of $<8 \%$ in conductivity. The selectivity test indicates that the fabricated hydrogen sensor had good selectivity, probably because of selective adsorption of $\mathrm{H}_{2}$ on the $\mathrm{Pd}$ nanoparticles.

The reproducibility of the hydrogen sensor was estimated from its response to $1 \%$ hydrogen. The performance was repeatable over 10 cycles of hydrogenation and dehydrogenation. The results reveal that the sensor had good reproducibility, with a relative standard deviation of $4.4 \%$, which indicates that the sensor structure was robust and its performance under different sensing conditions was consistent.

\section{Conclusions}

A PANI-rGO film doped with Pd nanoparticles was prepared for fabrication of a hydrogen sensor operating at room temperature. The nanocomposite was found to be 
highly sensitive to hydrogen. By taking advantage of the Pd-nanoparticle doping, both high sensitivity and excellent selectivity were attained along with hydrogen-gas-sensing capability with rapid response and recovery. A response time of $20 \mathrm{~s}$ and a recovery time $50 \mathrm{~s}$ were obtained when the sensor was exposed to $1 \mathrm{vol} \%$ hydrogen at $25{ }^{\circ} \mathrm{C}$. The large specific surface area of the mesoporous PANI-rGO composite and the catalytic activity of the Pd nanoparticles were the key factors of enhancement of the interaction between the hydrogen molecules and sensing surface, which led to the improvement in hydrogen-gas-sensing performance. The sensor can be used to detect hydrogen gas at room temperature with fast response and recovery time, which makes it promising for potential application in monitoring of safe hydrogen levels.

\section{Acknowledgments}

The authors wish to express their gratitude and appreciation for the National Science Foundation of China (51561006, 51461011, 51201042, 51261005, 51261005, 51461010, 51401059, 51361005, 51371060, and U1501242), the Guangxi Natural

Science Foundation (2013GXNSFBA019243, 2014GXNSFAA118318, 2014GXNSFBA118240, and 2015GXNSFAA139282), and the Innovation Project of Guangxi Graduate Education (YJCXS201565).

\section{References}

[1] Phan DT, Chung GS. Characteristics of resistivity-type hydrogen sensing based on palladium-graphene nanocomposites. Int J Hydrogen Energy 2014;39: 620-9.

[2] Pandey PA, Wilson NR, Covington JA. Pd-doped reduced graphene oxide sensing 
films for $\mathrm{H}_{2}$ detection. Sens Actuators B 2013;183:478-87.

[3] Phan DT, Chung GS. Effects of Pd nanocube size of Pd nanocube-graphene hybrid onhydrogen sensing properties. Sens Actuators B 2014;204: 437-44.

[4] Yu MH, Huang YC, Li C, Zeng YX, Wang W, Li Y, Fang PP, Lu XH, Tong YX. Building three-dimensional graphene frameworks for energy storage and catalysis. Adv Funct Mater 2015;25:324-30.

[5] Konwer S, Guha AK, Dolui SK. Graphene oxide-filled conducting polyaniline composites as methanol-sensing materials. J Mater Sci 2013; 48:1729-39.

[6] Phan DT, Chung GS. A novel Pd nanocube-graphene hybrid for hydrogen detection. Sens Actuators B 2014;199:354-60.

[7] Esfandiar A, Irajizad A, Akhavan O, Ghasemi S, Gholami MR. Pd- $\mathrm{WO}_{3} /$ reduced graphene oxide hierarchical nanostructures as efficient hydrogen gas sensors. Int J Hydrogen Energy 2014;39:8169-79.

[8] Phan DT, Chung GS. Reliability of hydrogen sensing based on bimetallic Ni-Pd/graphene composites. Int J Hydrogen Energy 2014;39:20294-304.

[9] Virji S, Kaner RB, Weiller BH. Hydrogen Sensors Based on Conductivity Changes in Polyaniline Nanofibers. J Phys Chem B 2006;110:22266-70.

[10] Sripada R, Parambath VB, Baro M, Nair SP N, Sundara R. Platinum and platinumeiron alloy nanoparticles dispersed nitrogen-doped graphene as high performance room temperature hydrogen sensor. Int $\mathrm{J}$ Hydrogen Energy 2015;40:10346-53.

[11] Zou YJ, Pisciotta J, Baskakov IV. Nanostructured polypyrrole-coated anode for sun-powered microbial fuel cells. Bioelectrochemistry 2010;79:50-6.

[12] Yang F, Xu MW, Bao SJ, Sun QQ. $\mathrm{MnO}_{2}$-assisted fabrication of PANI/MWCNT composite and its application as a supercapacitor. RSC Adv 2014;4:33569-73.

[13] Yu L, Gan MY, Ma L, Huang H, Hu HF, Li YJ, Tu Y, Ge CQ, Yang FF, Yan J. Facile synthesis of $\mathrm{MnO}_{2} /$ polyaniline nanorod arrays based on graphene and its electrochemical performance. Synth Met 2014;198:167-74.

[14] Sadek AZ, Wlodarski W, Kalantar-Zadeh K, Baker C, Kaner RB. Doped and 
dedoped polyaniline nanofiber based conductometric hydrogen gas sensors. Sens Actuators A 2007; 139:53-7.

[15] Wang PC, Dan YP, Liu LH. Effect of thermal treatment on conductometric response of hydrogen gas sensors integrated with $\mathrm{HCl}$-doped polyaniline nanofibers. Mater Chem Phys 2014;144:155-61.

[16] Cho SH, Lee JS, Jun J, Kim SG, Jang J. Fabrication of water-dispersible and highly conductive PSS-doped PANI/graphene nanocomposites using a high-molecular weight PSS dopant and their application in $\mathrm{H}_{2} \mathrm{~S}$ detection. Nanoscale 2014;6:15181-95.

[17] Liu C, Noda Z, Sasaki K, Hayashi K, Development of a polyaniline nanofiber-based carbon monoxide sensor for hydrogen fuel cell application. Int $\mathbf{J}$ Hydrogen Energy 2012;37:13529-35.

[18] Zhang Y, Wen FF, Jiang Y, Wang L, Zhou CH, Wang HG. Layer-by-layer construction of caterpillar-like reduced graphene oxide-poly(aniline-co-o-aminophenol)-Pd nanofiber on glassy carbon electrode and its application as a bromate sensor. Electrochim Acta 2014;115: 504-10.

[19] Sheng QL, Wang MZ, Zheng JB. A novel hydrogen peroxide biosensor based on enzymatically induced deposition of polyaniline on the functionalized graphene-carbon nanotube hybrid materials. Sens Actuators B 2011;160:1070-7.

[20] Zheng ZX, Du YL, Feng QL, Wang ZH, Wang CM. Facile method to prepare $\mathrm{Pd} /$ graphene-polyaniline nanocomposite and used as new electrode material for electrochemical sensing. J Mol Catal A: Chem 2012;353-354:80-6.

[21] Li J, Liu S, Yu JH, Lian WJ, Cui M, Xu W, Huang JD. Electrochemical immunosensor based on graphene-polyaniline composites and carboxylated graphene oxide for estradiol detection. Sens Actuators B 2013;188:99- 105.

[22] Qiu JD, Shi L, Liang RP, Wang GC, Xia XH. Controllable deposition of a platinum nanoparticle ensemble on a polyaniline/graphene hybrid as a novel electrode material for electrochemical sensing. Chem Eur J 2012;18:7950 -9.

[23] Lei W, Si WM, Xu YJ, Gu ZY, Hao QL. Conducting polymer composites with 
graphene for use in chemical sensors and biosensors. Microchim Acta $2014 ; 181: 707-22$.

[24] Srivastava S, Kumar S, Singh VN, Singh M, Vijay YK. Synthesis and characterization of $\mathrm{TiO}_{2}$ doped polyaniline composites for hydrogen gas sensing. Int J Hydrogen Energy 2011; 36: 6343-55.

[25] Nasirian S, Milani MH. Hydrogen gas sensing based on polyaniline/anatase titania nanocomposite. Int J Hydrogen Energy 2014; 39: 630-42.

[26] Li G, Li Y, Peng H, Qin Y. Synthesis and electrochemical performances of dispersible polyaniline/sulfonated graphene composite nanosheets. Synth Met $2013 ; 184: 10-5$.

[27] Sahoo S, Karthikeyan G, Nayak GC, Das CK. Modified graphene/polyaniline nanocomposites for supercapacitor application. Macromolecular Res 2012, 20: $415-21$.

[28] Li ZF, Zhang HY, Liu Q, Sun LL, Stanciu L, Xie J. Fabrication of high-surface-area graphene/polyaniline nanocomposites and their application in supercapacitors. ACS Appl Mater Interfaces 2013; 5: 2685-91.

[29] Al-Mashat L, Shin K, Kalantar-zadeh K, Plessis JD, Han SH, Kojima RW, Kaner RB, Li D, Gou Xl, Ippolito SJ., Wlodarski W. Graphene/polyaniline nanocomposite for hydrogen sensing, J Phys Chem C 2010;114: 16168-73.

[30] Yang Y, Li S, Yang W, Yuan W, Xu J, Jiang Y. In situ polymerization deposition of porous conducting polymer on reduced graphene oxide for gas sensor. ACS Appl Mater Interfaces 2014; 6:13807-14.

[31] Yuan W, Huang L, Zhou Q, Shi G. Ultrasensitive and selective nitrogen dioxide sensor based on self-assembled graphene/polymer composite nanofibers. ACS Appl Mater Interfaces 2014; 6: 17003-8.

[32] Lei W, Si W, Xu Y, Gu Z, Hao Q. Conducting polymer composites with graphene for use in chemical sensors and biosensors. Microchim Acta 2014; 181,707-22.

[33] Srivastava S, Sharma SS, Kumar S, Agrawal S, Singh M, Vijay YK. Characterization of gas sensing behavior of multi walled carbon nanotube 
polyaniline composite films. Int J Hydrogen Energy 2009; 34: 8444-50.

[34] Srivastava S. Sharma SS, Agrawal S, Kumar S, Singh M, Vijay YK. Study of chemiresistor type CNT doped polyaniline gas sensor. Synth Met 2010; 160: $529-34$.

[35] Kumar R, Varandani D, Mehta BR, Singh VN, Wen ZH, Feng XL, Müllen K. Fast response and recovery of hydrogen sensing in Pd-Pt nanoparticle-graphene composite layers. Nanotechnology 2011;22:275719 (7pp).

[36] Zengin H, Zhou WS, Jin JY, Czerw R, Smith DW, Echegoyen L,Carroll DL, Foulger SH, Ballato J. Carbon nanotube doped polyaniline. Adv Mater 2002; $14: 1480-3$

[37] Li L, Yan G, Wu J, Yu X, Guo Q, Ma Z, Huang Z. Preparation of polyaniline-palladium composite microflakes by one-step interface polymerization method. J Polym Res 2009; 16:421-6.

[38] Huang YF, Lin CW. Facile synthesis and morphology control of graphene oxide/polyaniline nanocomposites via in-situ polymerization process. Polymer 2012;53: 2574-82.

[39] Xiang CL, Cheng J, She Z, Zou YJ, Chu HL, Qiu SJ, Zhang HZ, Sun LX, Xu F. Fabrication and characterization of a novel nanoporous $\mathrm{Co}-\mathrm{Ni}-\mathrm{W}-\mathrm{B}$ catalyst for rapid hydrogen generation. RSC Adv 2015;5:163-6.

[40] Sekar P, Anothumakkool B, Kurungot S. 3D polyaniline porous layer anchored pillared graphene sheets: enhanced interface joined with high conductivity for better charge storage applications. ACS Appl Mater Interfaces, 2015;7:7661-9

[41]Kumar R, Malik S, Mehta BR. Interface induced hydrogen sensing in Pd nanoparticle/graphene composite layers. Sens Actuators B2015;209:919-26.

[42] Garcia-Aguilar J, Miguel-Garcia I, Berenguer-Murcia A, Cazorla-Amoros D. Single wall carbon nanotubes loaded with Pd and NiPd nanoparticles for $\mathrm{H} 2$ sensing at room temperature. Carbon 2014;66:599-611.

[43] Kaniyoor A, Imran Jafri R, Arockiadoss T, Ramaprabhu S. Nanostructured Pt decorated graphene and multi walled carbon nanotube based room temperature 
hydrogen gas sensor. Nanoscale 2009;1:382-6.

[44] Ou YJ, Si WW, Yu G, Tang LL, Zhang J, Dong QZ. Nanostructures of Pd-Ni alloy deposited on carbon fibers for sensing hydrogen. J Alloys Compd 2013;569:130-5.

[45] Yang X, Wang W, Xiong J, Chen L, Ma Y, ZnO:Cd nanorods hydrogen sensor with low operating temperature, Int J Hydrogen Energy 2015;40:12604-9. 


\begin{tabular}{|c|c|c|c|c|}
\hline \multirow[t]{2}{*}{ Samples } & Response (\%)/ & Response & Recovery & Ref. \\
\hline & (vol\%) $\mathrm{H}_{2}$ & time (s) & time (s) & \\
\hline $\mathrm{Pd}$-graphene composite & $7 / 0.1$ & 240 & 1200 & {$[1]$} \\
\hline $\mathrm{Ni} / \mathrm{Pd}$-graphene composite & $11 / 0.1$ & 180 & 720 & {$[8]$} \\
\hline $\mathrm{Pt} / \mathrm{Pd}$-graphene composite & $4 / 2$ & 2 & 60 & [35] \\
\hline $\mathrm{Ni}-\mathrm{Pd} / \mathrm{SWCNTs}$ & $10 / 0.5$ & 720 & 60 & [42] \\
\hline $\mathrm{Pt} / \mathrm{CNT}$ and graphene & $18 / 4$ & 1200 & 3600 & [43] \\
\hline $\mathrm{Pd}-\mathrm{Ni} /$ carbon fibers & $2 / 0.1$ & 720 & 60 & [44] \\
\hline $\mathrm{ZnO}: \mathrm{Cd}$ nanorods & $1.6 / 0.05$ & 43 & - & [45] \\
\hline $\mathrm{Pd}-\mathrm{PANI}-\mathrm{rGO}$ & $25 / 1$ & 20 & 50 & This work \\
\hline
\end{tabular}




\section{Figure captions}

Fig. 1. Schematic diagram of the preparation of the Pd-PANI-rGO nanocomposite.

Fig. 2. Schematic diagram of the fabrication of a hydrogen gas sensor.

Fig. 3. Schematic diagram of the gas-sensing setup.

Fig. 4. SEM images of (a) rGO, (b) PANI, (c) PANI-rGO, and (d) Pd-PANI-rGO.

Fig. 5. FTIR spectra of rGO, PANI, PANI-rGO, and Pd-PANI-rGO.

Fig. 6. XRD patterns of rGO, PANI, PANI-rGO, and Pd-PANI-rGO.

Fig. 7. $\mathrm{N}_{2}$ adsorption and desorption isotherms of rGO, PANI, PANI-rGO, and Pd-PANI-rGO nanoparticles.

Fig. 8. Gas-sensing responses of the rGO, PANI, PANI-rGO, and Pd-PANI-rGO thin films to $1 \%$ hydrogen at room temperature.

Fig. 9. Gas-sensing response of the Pd-PANI-rGO-based sensor to different concentrations of hydrogen at room temperature.

Fig. 10. Hydrogen response versus rGO concentration for the Pd-PANI-rGO-nanocomposite-based hydrogen sensor.

Fig. 11. Hydrogen response versus $\mathrm{K}_{2} \mathrm{PdCl}_{6}$ concentration for the Pd-PANI-rGO-nanocomposite-based hydrogen sensor.

Fig. 12. Hydrogen response versus temperature for the Pd-PANI-rGO-nanocomposite-based hydrogen sensor.

Fig. 13. Effect of temperature on the response time and recovery time of the Pd-PANI-rGO-nanocomposite-based hydrogen sensor.

Fig.14. Response of the Pd-PANI-rGO-nanocomposite-based sensor to various gases. 


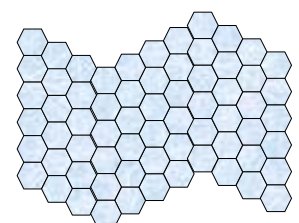

GO

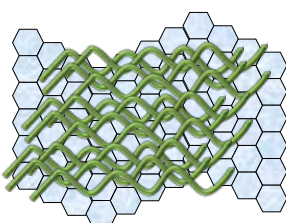

N

PANI

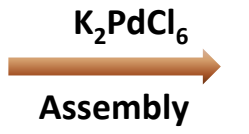

Pd

Fig.1. Schematic diagram of the preparation of the Pd-PANI-rGO nanocomposite. 


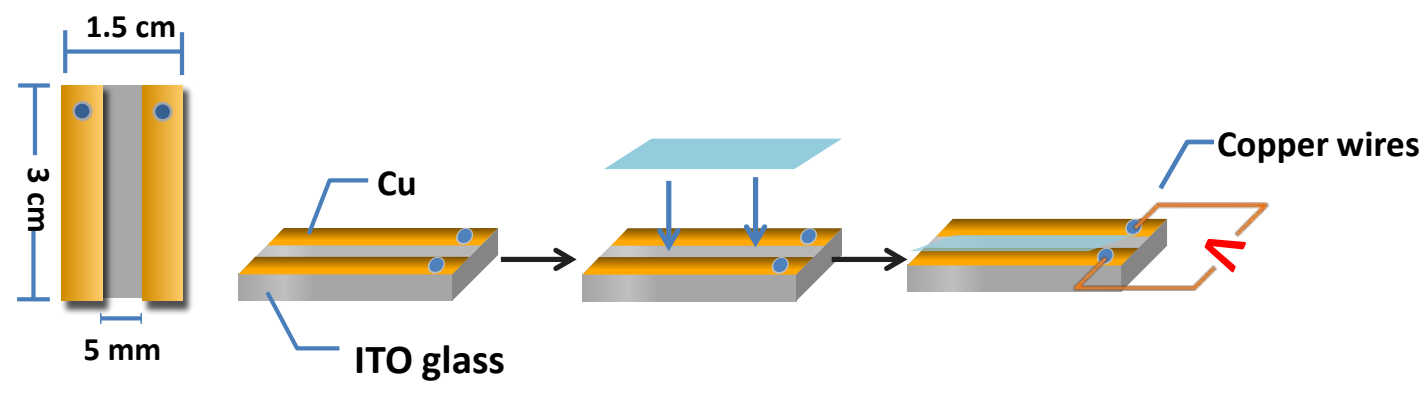

Fig. 2. Schematic diagram of the fabrication of a hydrogen gas sensor. 


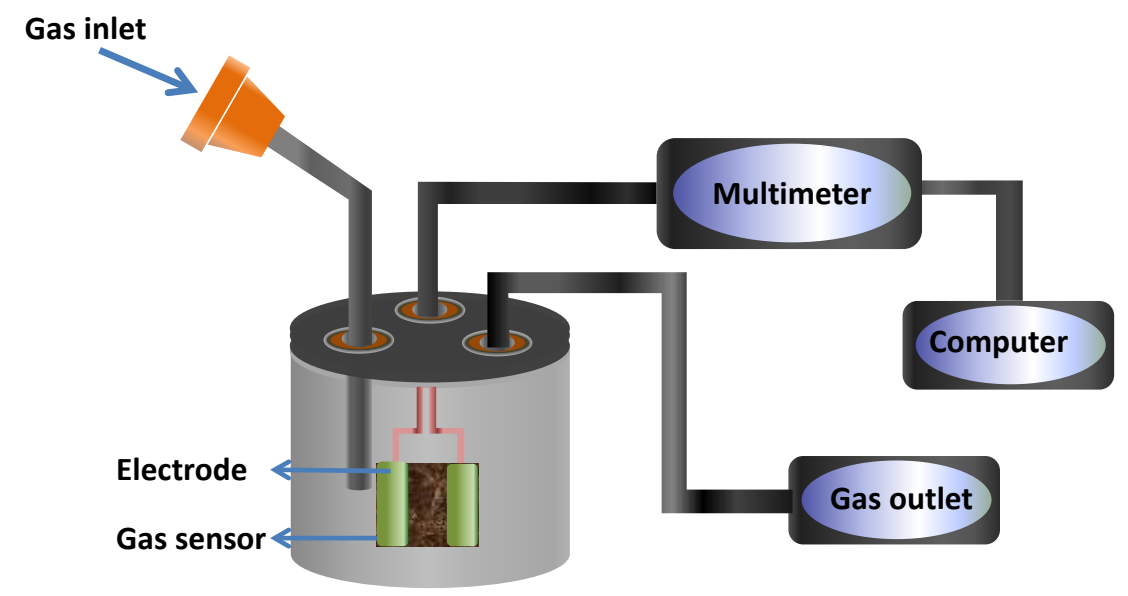

Fig. 3. Schematic diagram of the gas-sensing setup. 

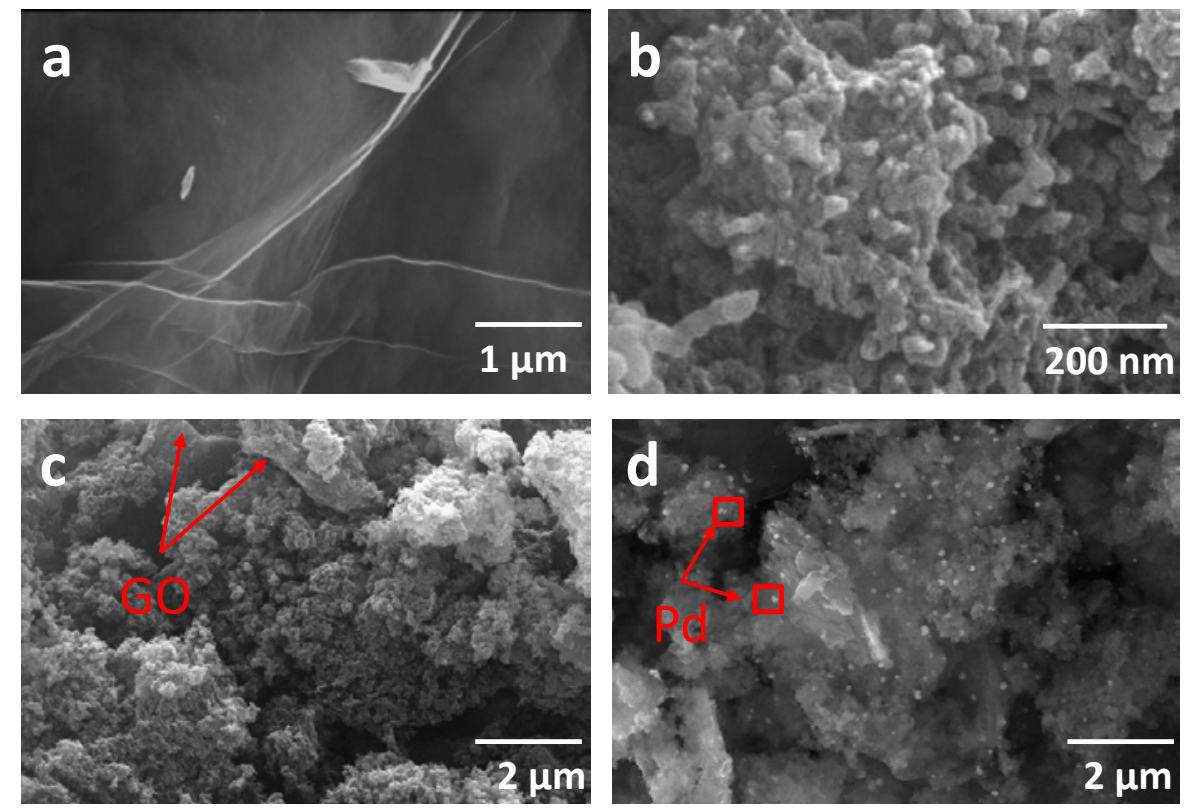

Fig. 4. SEM images of (a) rGO, (b) PANI, (c) PANI-rGO, and (d) Pd-PANI-rGO. 


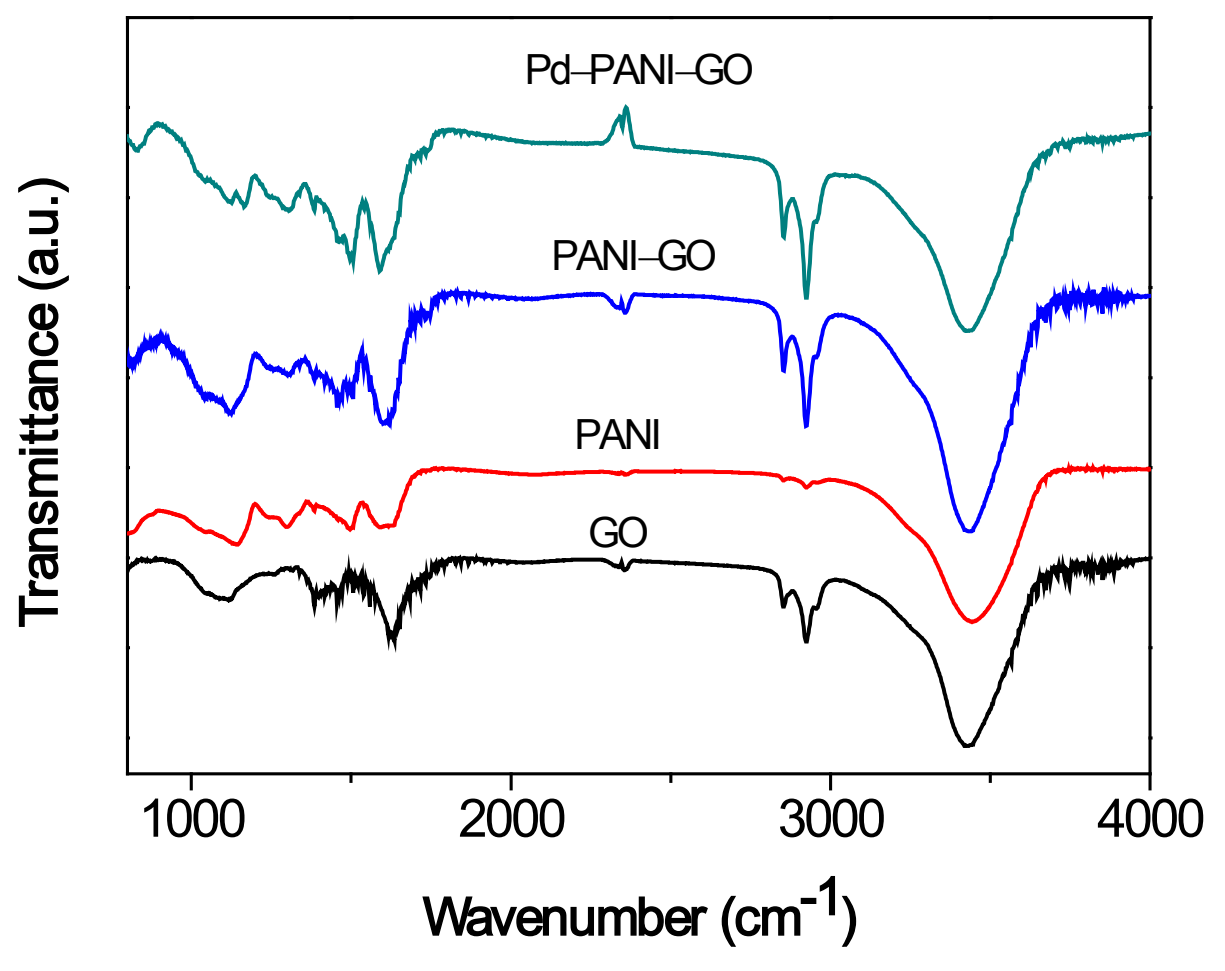

Fig. 5. FTIR spectra of rGO, PANI, PANI-rGO, and Pd-PANI-rGO. 


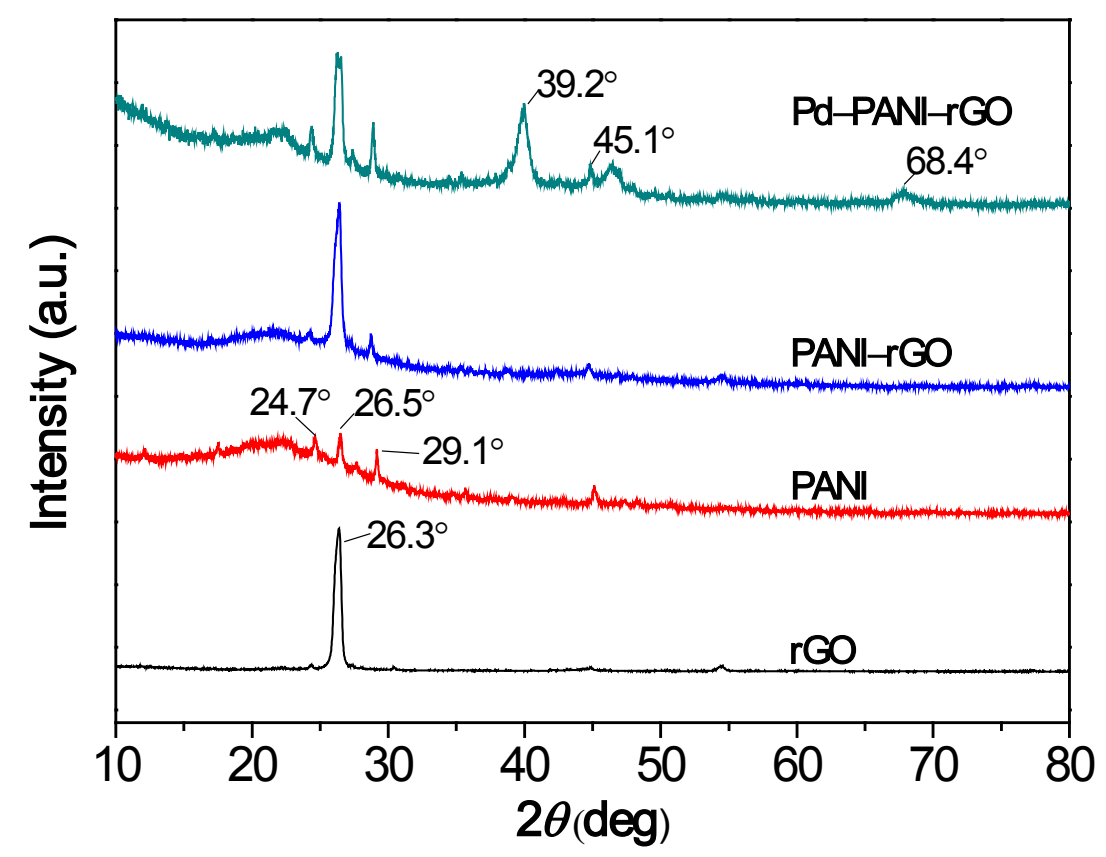

Fig. 6. XRD patterns of rGO, PANI, PANI-rGO, and Pd-PANI-rGO. 


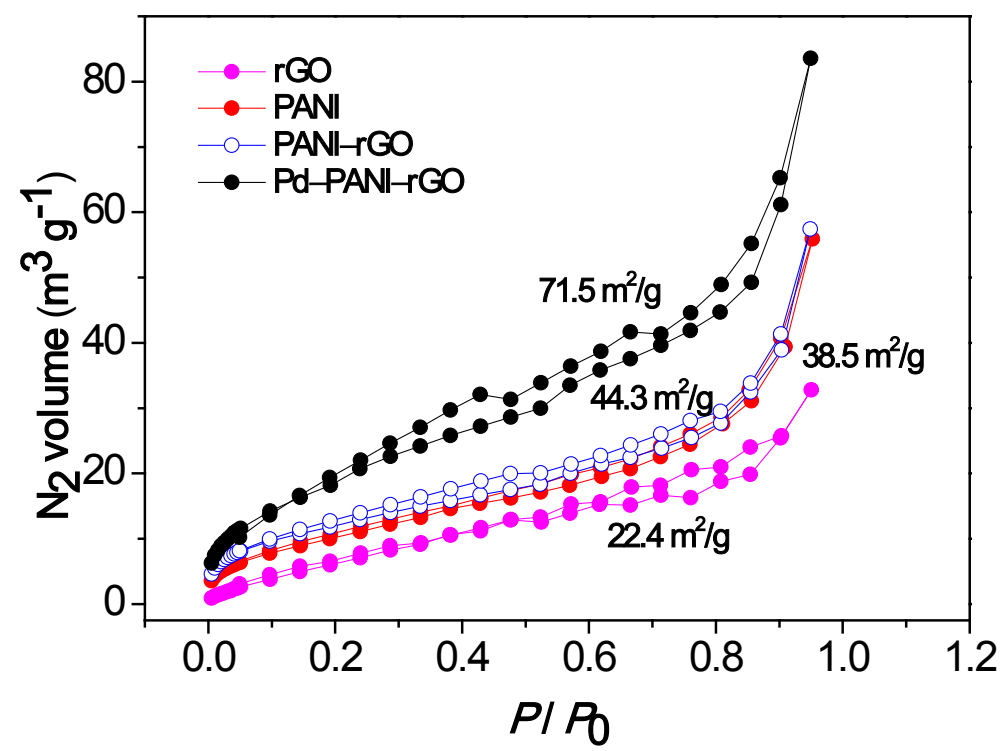

Fig. 7. $\mathrm{N}_{2}$ adsorption and desorption isotherms of rGO, PANI, PANI-rGO, and Pd-PANI-rGO nanoparticles. 


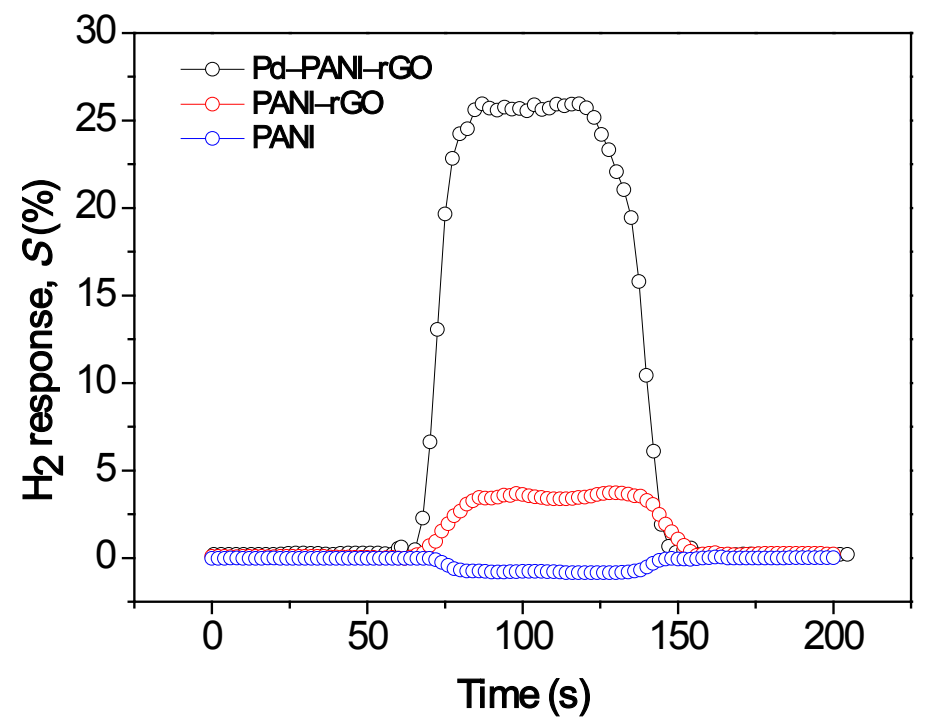

Fig. 8. Gas-sensing responses of the rGO, PANI, PANI-rGO, and Pd-PANI-rGO thin films to $1 \%$ hydrogen at room temperature. 

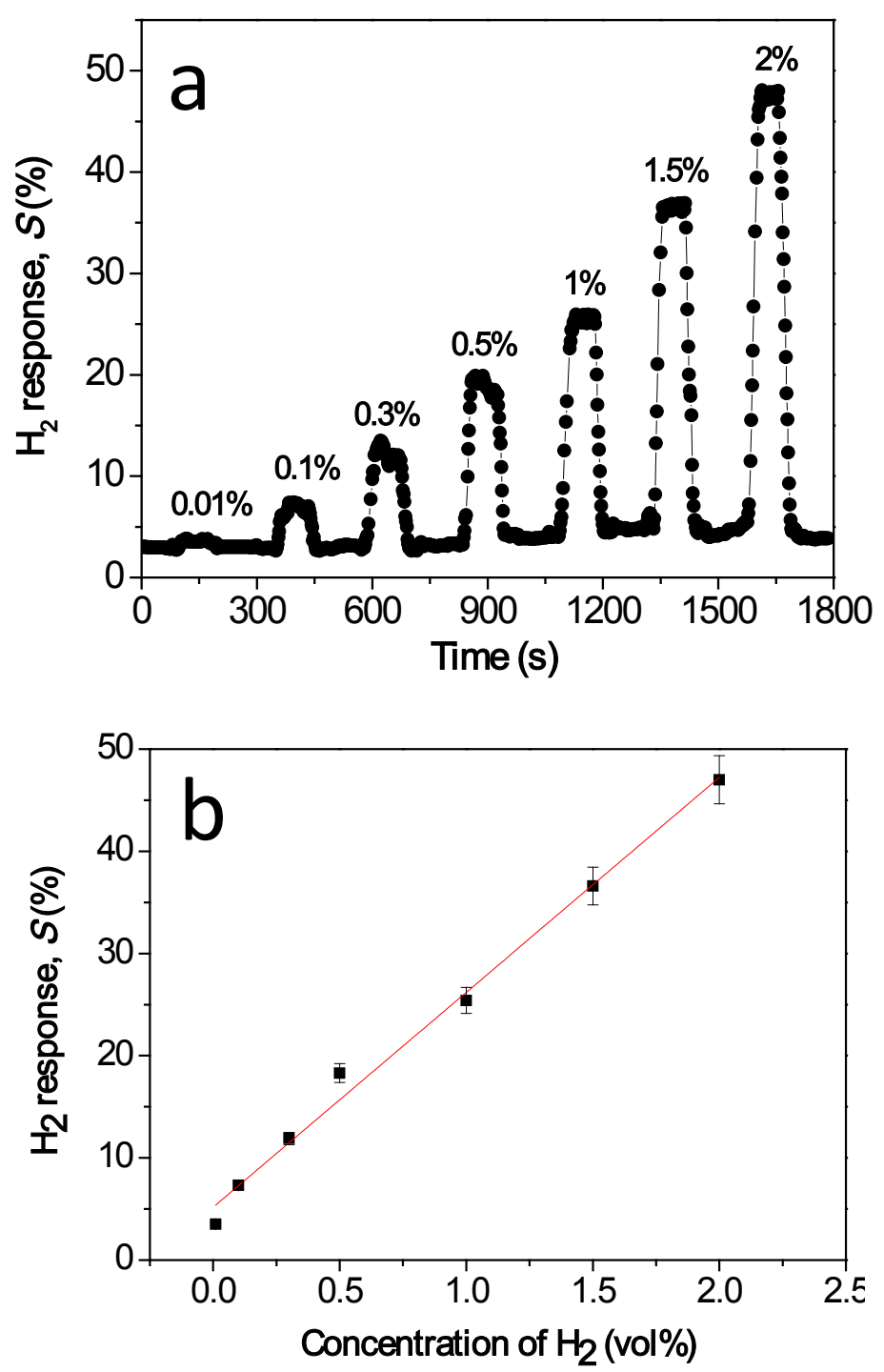

Fig. 9. Gas-sensing response of the Pd-PANI-rGO-based sensor to different concentrations of hydrogen at room temperature. 


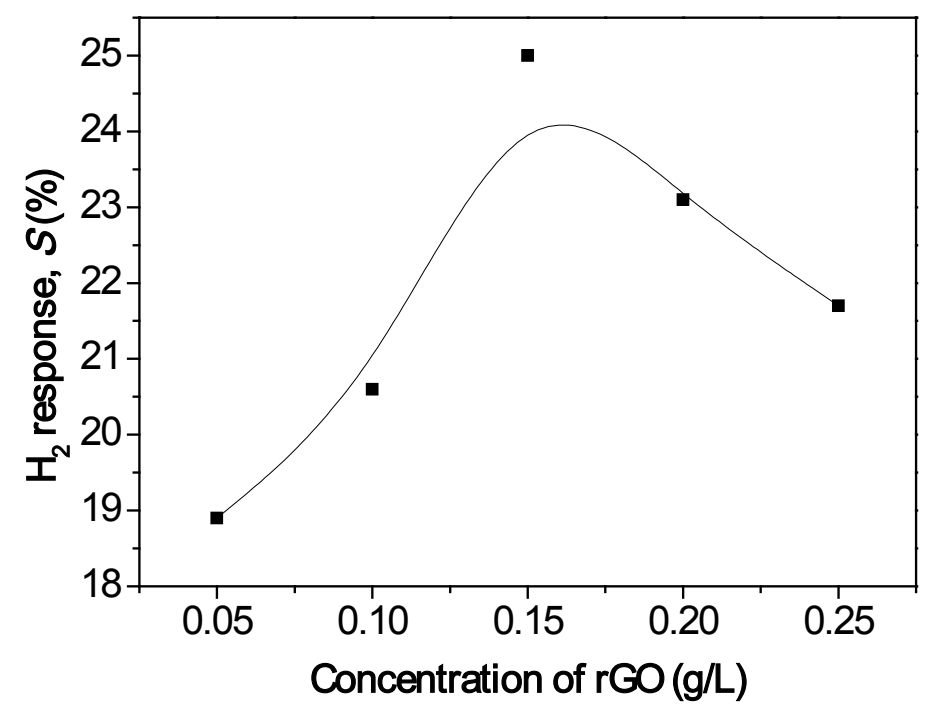

Fig. 10. Hydrogen response versus rGO concentration for the Pd-PANI-rGO-nanocomposite-based hydrogen sensor. 


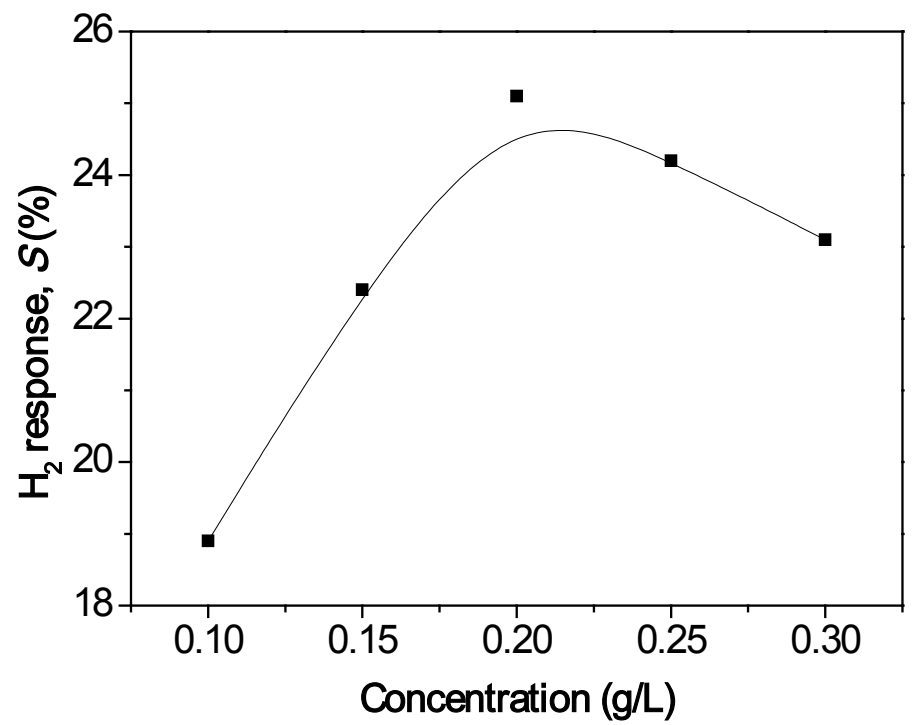

Fig. 11. Hydrogen response versus $\mathrm{K}_{2} \mathrm{PdCl}_{6}$ concentration for the Pd-PANI-rGO-nanocomposite-based hydrogen sensor. 


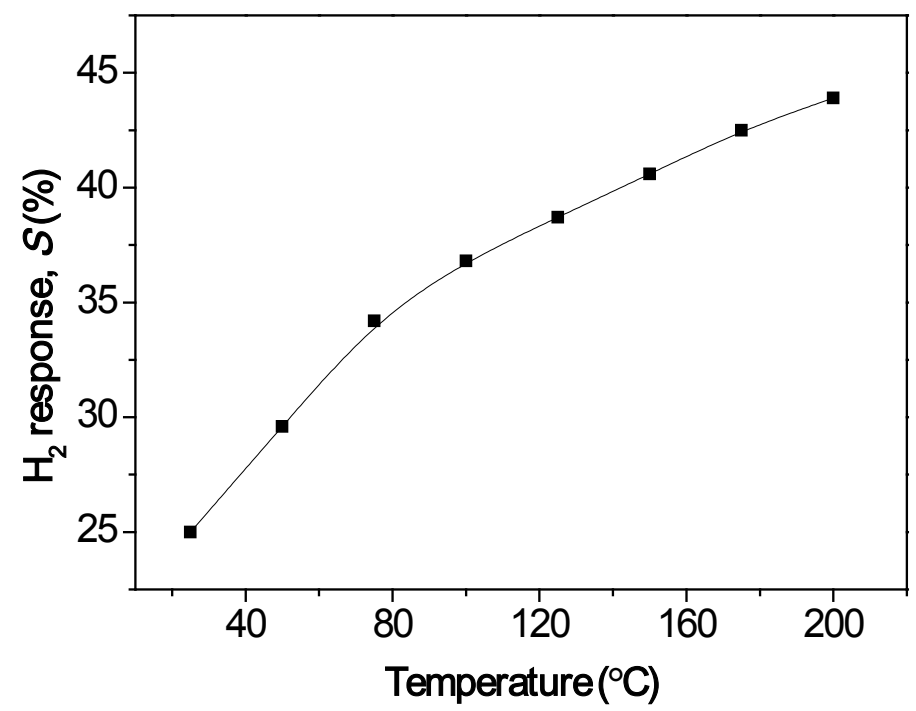

Fig. 12. Hydrogen response versus temperature for the Pd-PANI-rGO-nanocomposite-based hydrogen sensor. 


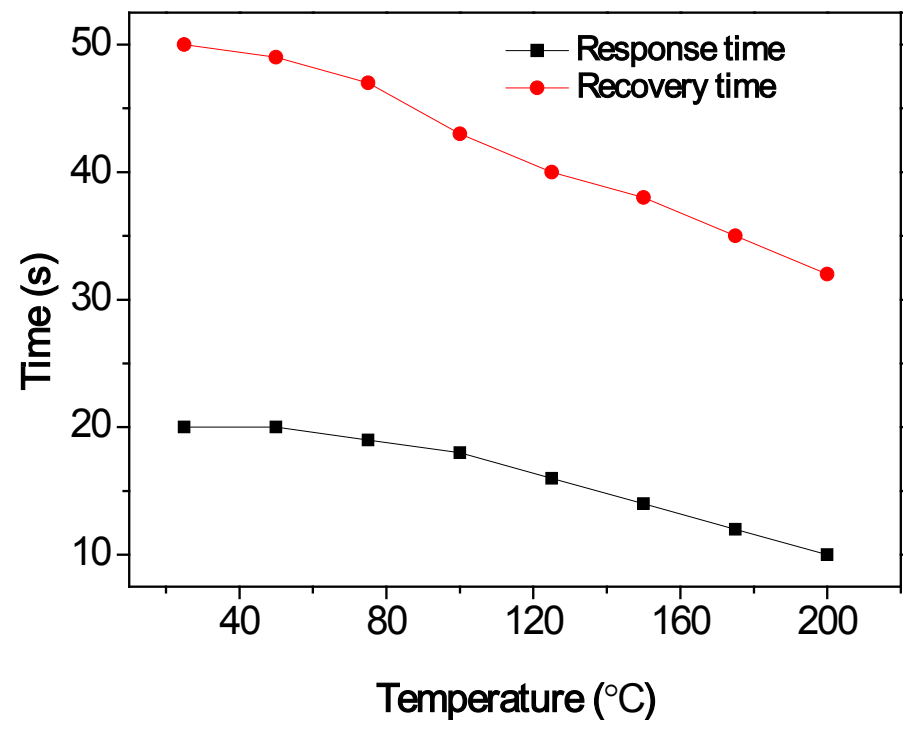

Fig. 13. Effect of temperature on the response time and recovery time of the Pd-PANI-rGO-nanocomposite-based hydrogen sensor. 


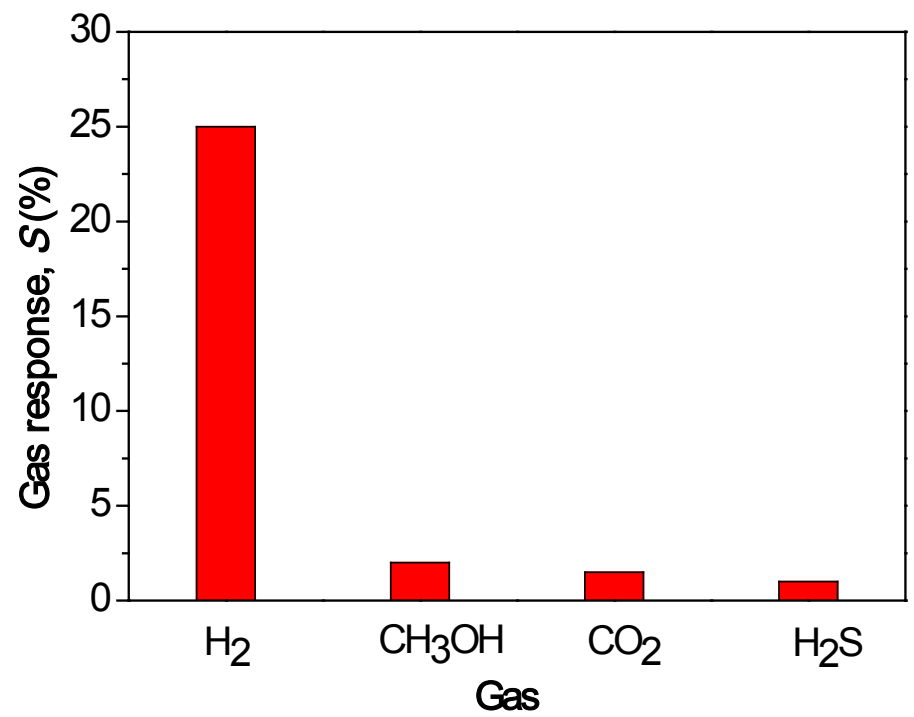

Fig.14. Response of the Pd-PANI-rGO-nanocomposite-based sensor to various gases. 